\title{
La professionnalité du métier d'enseignant de FLE-S au carrefour de discours, identités et savoirs
}

\author{
Jenny Moreno ${ }^{l}$ \\ ${ }^{l}$ Docteure en Sciences du langage, Université de Nantes, France.
}

\begin{abstract}
Résumé. Les constructions discursives autour des savoirs, du savoir-être et des savoir-faire des futurs enseignants de FLE-S permettent d'identifier les représentations identitaires qui se construisent sur leur avenir professionnel. Le but de cette étude est de connaître les images identitaires qui intègrent la professionnalité des étudiants natifs et non-natifs, suivant une formation de Master FLE-S à l'Université en France. Les postulats théoriques et méthodologiques nous permettant de parvenir à ce but et de traiter les trois types de discours choisis pour ce travail, à savoir, les discours définitionnels, les discours d'experts et ceux des étudiants sont d'une part, la Sémantique des Possibles Argumentatifs qui nous permet de faire la reconstruction de la signification lexicale de la professionnalité à l'interface de représentations sémantiques et discursives qui s'associent au sens premier mot et d'autre part, la Didactique Analytique nous permettant de faire une étude exploratoire des savoirs, des savoirs d'action et des images qui se mobilisent autour de la formation personnelle, académique et professionnelle des étudiants. L'exploration conjointe des représentations produites dans et par le discours, l'association des savoirs et l'activation d'images identitaires nous amènent à légitimer, de par nos résultats, le besoin d'étudier le regard que les étudiants portent sur leur formation.
\end{abstract}

\begin{abstract}
The professionalism of the FFL-FSL teaching profession at the junction of discourse, identities and knowledge. The discursive construction of knowledge, knowing about knowledge to do, and knowing how to be in future teachers of FFL-FSL allows to identify the identitary representations that they build on their professional future. The aim of this study is to know the identitary images that integrate the professionalism of a native and non-native students, following a Master's degree in FFL-FSL at the French University. The theoretical and methodological postulates allowing us to achieve this aim and to deal with the three types of discourse chosen for this work, namely, definitional discourse, expert discourse and student discourse, are on the one hand, the Semantics of Argumentative Probabilities which allows us to reconstruct the lexical meaning of professionalism at the interface of semantic and discursive
\end{abstract}

\footnotetext{
1jekamo@hotmail.com
} 
representations that are associated in the first word sense and on the other hand, Analytical Didactics which allows us to make an exploratory study of the knowledge, action knowledge and images that are involved around the personal, academic and professional training of the students. The shared exploration of representations produced in and by the discourse, the association of knowledge and the activation of identity images lead us to legitimize, through our results, the need to study the students' view of their formation.

\section{Introduction}

Le futur enseignant de Français langue étrangère et seconde participe constamment à un processus de reconstruction de son identité personnelle, collective et professionnelle. Afin de repérer les mécanismes qui contribuent à cette construction, voire reconstruction identitaire, nous allons faire dans une première partie une analyse sémantique et discursive de l'entité lexicale «professionnalité ». Selon Barbier, la professionnalité est « l'ensemble des savoirs, des compétences et des dispositions mobilisées par un individu dans l'exercice d'une activité professionnelle spécifique » (Barbier, 1996 :53).

D'un point de vue historique, le terme a été utilisé en France autour des années 70 et apparaît dans une ambiance de crise identitaire du métier enseignant. Selon le Rapport de Bancel (1989), le plus important dans l'histoire du mot, la construction de la professionnalité enseignante est strictement liée au concept de «professionnalisation». Dans ce rapport, la professionnalisation est définie comme « le moyen de revalorisation sociale du métier enseignant» (Bancel, 1989) et la concrétisation de ce processus de professionnalisation est ainsi la construction d'une professionnalité enseignante. Pourquoi avons-nous eu recours à l'étude dudit concept ? Parce qu'il va au-delà de la valeur de l'action dans un métier et " devient un outil pour l'action et pour la recherche permettant d'appréhender différemment les conditions individuelles et collectives de la réalisation du travail » (Barbier et al, 1996 : 168), dans notre cas, des futurs enseignants de Français.

Inspirés des travaux menés par Galatanu (2014) et Barbier (1998, 2004), nous nous intéressons aux discours déclaratifs des futurs enseignants de FLE-S portant sur la professionnalité. Ceux-ci véhiculent des systèmes de valeurs, voire une identité discursive qui permet de reconstruire leur identité comme futurs enseignants. Le concept de la professionnalité fait ainsi partie des éléments majeurs qui contribuent à l'édification d'une ou des identités dans le cadre des métiers de l'enseignement.

La deuxième partie de notre travail consiste à explorer au moyen des postulats qui défendent la perspective applicationniste de la Didactique Analytique, l'existence d'une dimension instrumentale et rationnelle de la professionnalité dans les discours mobilisés par le public en question. L'instrumentalité de la professionnalité s'associe à la production mécanique de procédés, parfois non-volontaires et dans d'autres cas, imposés qui amènent l'étudiant à finaliser une formation sans entamer une projection professionnelle réfléchie et consciente. La dimension rationnelle de la professionnalité s'attache plutôt aux stratégies volontaires et réfléchies de mise en action qui conduisent l'étudiant à se professionnaliser grâce à son interaction avec le terrain socio-professionnel qui l'entoure.

Pour ce faire, nous devons donc procéder à l'analyse des images identitaires liées aux savoirs et aux savoirs d'action mobilisés par les futurs diplômés du Master FLE-S formés en 
France; celles-ci contribueront bien évidemment à identifier les mécanismes à travers lesquels les étudiants construisent leur professionnalité.

\section{Dispositif de la SPA : repères théoriques, application méthodologique et analyses}

La Sémantique des Possibles Argumentatifs (SPA) (Galatanu, 2009a, 2009b) est un modèle théorique et méthodologique qui a pour objectif d'élaborer la description de la signification lexicale des mots ou entités lexicales à partir de deux approches: l'approche de la signification linguistique, inspirée des fondements théoriques de la sémantique et de l'Analyse du Discours (AD) et l'approche du sens discursif. La première représente le processus de construction et reconstruction de la signification lexicale, pouvant rendre compte de son cinétisme sémantique. La deuxième soutient que le sens des énoncés discursifs inscrit un potentiel axiologique et donc argumentatif dans la signification des entités linguistiques. Ce potentiel axiologique se présente sous forme d'associations de mots et se caractérise par son ancrage culturel et, notamment, évolutif (Galatanu, 2009a).

Compte tenu de ces deux approches, Galatanu propose un dispositif de la signification présenté en termes de Noyau (N), de Stéréotypes (Sts), de Possibles Argumentatifs (PA) et d'une forme de manifestation discursive, dénommée "Déploiements Argumentatifs » (DA). Elle s'inspire principalement des travaux sur la signification des mots, menés par Putnam $(1975,1990)$ et les met en filiation avec les postulats de la sémantique argumentative (Anscombre, Ducrot, 1994 ; Ducrot, Carel, 2005). Au sein du dispositif, le Noyau est défini comme l'ensemble d'éléments stables de la signification lexicale (Galatanu, 2009b). Ces éléments sont extraits des discours dictionnairiques, voire encyclopédiques ayant pour but la définition des mots. Les Stéréotypes représentent l'ensemble ouvert d'associations du noyau avec d'autres représentations que la linguiste va appeler «des blocs d'argumentation interne" (Galatanu, 2009b) au mot. Les Possibles Argumentatifs sont des séquences discursives déployant l'association du mot avec un élément de son stéréotype. Par exemple, selon nos données, le PA «Professionnalité DC métier » est plus récurrent, attestant ainsi de la saillance du stéréotype «métier » toujours associé à la professionnalité. La dernière strate du dispositif est celle des DA ou occurrences discursives. Celles-ci se situent à l'interface de la langue et du discours et relèvent des séquences argumentatives apparues dans des énoncés. À l'issue des quatre niveaux d'analyse de la signification, nous allons obtenir la reconstruction sémantique de la professionnalité. Cela sera traité sur le plan discursif, c'est-à-dire à partir du discours des étudiants suivant un parcours de Master FLE-S à l'Université en France.

Le modèle de description des significations lexicales proposé par la SPA tient également compte d'une approche holistique, associative et encyclopédique. Il est associatif et holistique car la SPA défend l'idée dans laquelle, les stéréotypes figés à un mot « représentent des associations, dans des blocs de signification argumentative (relation posée comme une «relation naturelle » : cause-effet, symptôme phénomène, but moyen, etc.) des éléments du noyau avec d'autres représentations sémantiques » (Galatanu, 2006 : 94) et ces associations sont normalement stables et ont un ancrage culturel favorisant l'inscription de "nouveaux éléments relevant du contexte culturel et contextuel dans la signification des mots» (Galatanu, 2006 : 95). Le modèle est aussi encyclopédique «car tous les aspects de notre connaissance de l'entité en jeu contribuent au sens de l'expression qui la désigne » (Galatanu, $2006: 95)$.

En tenant compte des strates et des approches, notre démarche méthodologique consiste à reconstruire la signification lexicale de la «professionnalité » à partir des dictionnaires 
(car il est un outil lexical partagé qui met en lien la langue et la culture pour définir les mots et les définitions lexicographiques font apparaître les éléments stables du noyau des mots), des définitions d'experts et du discours des étudiants. Notre contexte de travail est ainsi intégré par un public d'étudiants natifs et non-natifs inscrits dans un programme de Master FLE-S de cinq universités françaises (année scolaire 2015-2016) : Université d'Angers, Université de Tours, Université de Rennes 2, la Sorbonne Paris et l'Université de Nantes. Ces établissements d'enseignement supérieur ont été choisis car ils ont un grand nombre d'effectifs dans le programme de Master par rapport à d'autres universités en France.

Suivant le dispositif de la SPA proposé par Galatanu, nous procédons à la construction du discours lexicographique de la professionnalité. Pour entamer la construction discursive de la signification lexicale de la professionnalité, nous avons eu recours aux définitions publiées dans quatre dictionnaires italiens, à savoir, Il dizionario della lingua italiana, Grande Dizionario della lingua italiana moderna, De Mauro il dizionario della lingua italiana et Dizionario di italino en ligne Treccani. Nous devons nous appuyer sur des dictionnaires italiens car les origines du mot professionnalité ne naissent pas avec la langue française, elles remontent à l'époque de luttes des travailleurs italiens et donc, ce mot n'apparaît pas dans les dictionnaires encyclopédiques français anciens ou modernes.

À partir des définitions apparues dans ces dictionnaires, nous avons identifié, dans la colonne gauche, le noyau du mot en étude, et dans la colonne droite, les stéréotypes qui lui sont associés attribués par les dictionnaires (Moreno, 2013).

\section{Qualité/condition personnelle de S \\ [+humain] pour faire X [activité/métier]}

\section{S avoir les capacités pour exercer $\mathrm{X} \quad$ DC exercice}

\section{DC}

\section{S pouvoir faire $\mathrm{X}$}

DC avoir un haut degré

DC métier

DCactivité

Figure 1. Représentation conceptuelle de la professionnalité au regard du discours dictionnairique.

D'après de cette première construction nous identifions le caractère opérationnel du terme, c'est-à-dire que le sujet $<$ humain $>$ mobilise des moyens pour développer un certain 
métier. Ensuite, nous relevons des stéréotypes qui s'associent aux éléments du noyau : < DC exercice $>,<$ DC avoir un haut degré $>,<$ DC métier $>,<$ DC activité $>$.

Les stéréotypes présentés dans la colonne de la droite témoignent du lien existant entre le métier et sa pratique. Par conséquent, nous ne pouvons pas continuer à illustrer ce modèle si nous ne situons pas le concept de professionnalité au sein du contexte français, celui-ci étant au cœur de notre recherche. Pour cela, nous avons eu recours aux discours de quatre experts français ayant publié des recherches en enseignement et autour du mot étudié.

Les auteurs étudiés sont Bourdoncle et Mathey-Pierre (1995), Barbier (1996) et Lang (1999). En prenant en compte leurs travaux, nous présentons le noyau qui suit et nous le mettons en lien avec des stéréotypes du mot encore une fois présentés dans la partie droite de la figure ci-dessous :

\section{STEREOTYPES IDENTIFIES}

Ensemble de savoirs/compétences/capacités

$\begin{array}{cl}\text { mobilisés par S [+ humain] pour faire X [activité } & \text { DC exercice d'acquisition de savoirs } \\ \text { professionnelle] } & \text { DC reconstruction biographique de l'individu } \\ \text { DC } & \end{array}$

$X$ pouvoir être <instrumentale $>$ et $\langle$ rationnelle $>$

DC acte de travail

DC entreprise

DC

Développement professionnel de S pour faire

évoluer X

DC se soumettre à des épreuves

DC

Valorisation de S par X

DC professionnalisation

DC valorisation du service rendu

Figure 2. Représentation conceptuelle de la professionnalité au regard du discours des experts.

Du point de vue des experts, la professionnalité recouvre beaucoup plus d'éléments de l'exercice d'un métier et tient compte du besoin d'implication du Sujet (S) pour rendre son activité professionnelle reconnue et mieux valorisée. Compte tenu des représentations construites à partir des discours des dictionnaires et des experts, nous proposons maintenant de faire une reconstruction sémantique de l'entité lexicale en question. Cela nous permettra d'observer l'ancrage culturel et évolutif que porte le mot professionnalité dans le contexte social et éducatif français. 


\author{
Noyau dur \\ Mobilisation des qualités humaines de S [+ \\ humain] pour faire $\mathrm{X}$ [activité professionnelle] \\ DC \\ savoirs/compétences/capacités de S [+ humain] \\ pour faire évoluer $\mathrm{X}<$ instrumentale $>$ et \\ $<$ rationnelle $>$ \\ DC \\ Développement personnel et professionnel de S \\ [+ humain] \\ DC \\ Valorisation de $\mathrm{X}$ par $\mathrm{S}$
}

Figure 3. Construction sémantique de la professionnalité à l'égard des corpus définitionnels.

Cette analyse sémantique nous amène à évoquer quelques conclusions préliminaires. Parmi elles, la place du sujet-acteur joue un rôle fondamental dans l'exercice de l'activité professionnelle; le Sujet est toujours en état de construction, il est capable de s'adapter à son environnement socioprofessionnel. À travers son savoir-être, ses savoirs savants et savoirs pratiques, il cible des actions qui contribuent à affirmer son identité professionnelle et à mettre en valeur son investissement personnel. Nous constatons aussi qu'il y a un cheminement inverse au niveau des représentations sémantiques, ce que Galatanu (2005: 193) considère comme le résultat d'un phénomène de contagion sémantique. Ceci s'explique parce qu'il y a des incidences de la représentation du discours lexicographique des dictionnaires sur le discours définitionnel des experts et vice-versa.

\title{
1.1 Reconstruction de l'entité lexicale professionnalité à l'égard des discours des étudiants
}

En partant du noyau sémantique édifié plus haut et du protocole de la théorie spéaienne, nous allons identifier à l'aide d'un questionnaire expérimental les DA de la professionnalité, ceux-ci nous permettront de constater quels sont les Sts et les PA qui leur sont associés. L'application du questionnaire a pour but de rendre visibles les définitions, les associations, les conceptions et, dans l'ensemble, les images identitaires évoquées par un public étudiant, voire des futurs enseignants, autour de la professionnalité. Le questionnaire est divisé en trois parties : dans la première partie, le public enquêté doit associer librement des mots à la professionnalité ; dans la deuxième partie, il doit évaluer des associations proposées en indiquant si celles-ci sont toujours, parfois ou jamais acceptées (avis personnel) ; finalement, il doit définir avec leurs propres mots ce qu'est la professionnalité.

Pour mener à bien notre étude, nous avons analysé un total de 120 questionnaires (cependant seulement 86 avaient été entièrement répondus) pendant une durée de deux mois dans l'année universitaire 2015-2016 (année de préparation de la thèse doctorale). Les cent vingt questionnaires initialement analysés correspondent au nombre d'informateurs inscrits en M1 et M2, et ont été enquêtés dans l'ensemble des universités : l'Université de Nantes avec 36 effectifs, l'Université de Tours avec 16 inscrits, l'Université d'Angers avec 27 étudiants, l'Université de Rennes 2 avec 18 effectifs et l'Université Sorbonne Paris 3 
avec 23 inscrits. Sur cette totalité, $60,8 \%$ des informateurs sont inscrits en M1 et 39,5\% en M2 ; 77 d'entre eux sont originaires des pays francophones et le $43 \%$ restant sont de nationalité étrangère.

Avec le but d'illustrer la construction du modèle au niveau de ses stéréotypes, des possibles argumentatifs et des déploiements argumentatifs, et pour en tirer les éléments saillants de ces strates, nous nous sommes appuyés sur l'usage du service de base du Logiciel Le Trameur. Ce logiciel, proposé par des chercheurs à l'Université Sorbonne Paris 3 , permet entre autres de comptabiliser le nombre de mots qui se répètent le plus dans les corpus discursifs insérés sur leur système. Après avoir traité informatiquement les mots apparus dans nos corpus, les stéréotypes qui s'activent principalement à la professionnalité sont: travail (10 occurrences), professionnel (9 occurrences), compétences (8 occurrences), métier (6 occurrences), savoir-faire (5 occurrences), formation (4 occurrences), capacité ( 4 occurrences), qualité ( 4 occurrences), maîtrise (3 occurrences), cadre professionnel (3 occurrences), exercice ( 2 occurrences) et savoir ( 2 occurrences). Ces stéréotypes ou blocs d'argumentation interne correspondent aux éléments inscrits dans le noyau sémantique du mot, qui a été reconstruit à partir de discours lexicographiques et d'experts auparavant.

L'évaluation de la deuxième partie du questionnaire (validation faite par les étudiants sur 15 associations proposées à l'égard de l'ancrage langagier et culturel du mot) nous permet d'obtenir les PA de notre unité lexicale. Ceux-ci ont été présentés sous forme d'associations pouvant être toujours, parfois ou jamais acceptées par les étudiants. Les séquences qui ont été acceptés par la majorité des étudiants sont $<$ Professionnalité DC exercice d'acquisition de savoirs professionnels $>$, $<$ Professionnalité DC entreprise $>$, $<$ Professionnalité DC métier $>$, $<$ Professionnalité DC acte de travail $>$, celles-ci prouvent que la professionnalité est toujours liée à l'agir et donc à l'acquisition de savoirs professionnels.

L'évaluation des associations $<$ Professionnalité DC terme instable $>,<$ Professionnalité PT chômage $>$ et $<$ Professionnalité DC problématique de qualification du métier $>$, de son côté, rend possible l'existence du chômage et d'une problématique socioprofessionnelle autour de la valorisation du métier d'enseignant de FLE-S. Selon ces résultats, chez nos informateurs, la professionnalité ne tient pas forcément compte du parcours biographique du sujet. Cette affirmation se justifie à travers le pointage de l'association $<$ Professionnalité DC reconstruction biographique de l'individu $>$ (parfois $=27$ réponses, jamais $=23$ réponses). $\mathrm{Au} \mathrm{vu}$ des pourcentages obtenus, les associations $<$ Professionnalité DC se soumettre à des épreuves $>,<$ Professionnalité DC discours $>,<$ Professionnalité DC professionnalisation $>$, $<$ Professionnalité DC avoir un haut degré $>,<$ Professionnalité DC valorisation du service rendu $>,<$ Professionnalité DC croissance $>$ et $<$ Professionnalité DC faire reconnaître le patrimoine commun du groupe social $>$, sont partiellement acceptées par nos informateurs et sont ainsi conformes au protocole sémantique du mot.

Nous pouvons tout de même associer la professionnalité à deux axes supplémentaires. Par exemple, les séries <Professionnalité DC discours $>$, <Professionnalité DC professionnalisation $>$ et $<$ Professionnalité DC faire reconnaître le patrimoine commun du groupe social $>$ relèvent chez les étudiants de l'influence des discours des acteurs socioéconomiques dans la construction de la professionnalité. De même, l'approbation des déploiements argumentatifs <Professionnalité DC se soumettre à des épreuves>, $<$ Professionnalité DC croissance $>,<$ Professionnalité DC valorisation du service rendu $>$ et 
$<$ Professionnalité DC avoir un haut degré>, met en relief l'existence d'une subjectivité notamment ancrée à l'esprit de compétition, à la formation permanente comme stratégie de croissance, à la reconnaissance et à la revalorisation du métier.

Concernant les DA, c'est-à-dire les occurrences apparues dans la troisième partie du questionnaire (définition libre attribuée au mot), nous avons identifié que la professionnalité chez des acteurs de l'enseignement tente de passer d'une dimension instrumentale à une dimension rationnelle (Lang, 2005). En d'autres termes, les savoirs convoqués autour de la définition du mot ont un caractère opératoire, voire instrumental, mais aussi réflexif, voire rationnel.

Afin de mieux comprendre ce passage, nous avons classifié les DA en trois groupes, d'abord ceux qui sont liés au savoir-agir, ensuite aux savoir-faire et finalement au savoirêtre. Concernant le savoir-agir, nous avons associé la professionnalité aux mots : investir dans la profession, concurrence professionnelle, faute professionnelle ; pour le savoir-faire, les mots à associer sont : processus d'apprentissage, former, capable d'assurer un métier ; pour le savoir être, les entités saillantes sont : bon esprit, ouverture d'esprit, amour.

Comme nous l'avons constaté dans les PA, les premiers résultats indiquent que la représentation de la professionnalité relève d'une subjectivité qui n'est pas celle des futurs enseignants de FLE-S, mais celle des acteurs socioéconomiques. Cela signifie que la plupart de leurs manifestations discursives ont été associées au savoir-agir et au savoir-faire (la pratique) dans la profession. De même, les étudiants ont associé des DA attachés davantage aux savoirs, voire l'acquisition de savoirs, en priorisant leur participation à une formation permanente. Enfin, les étudiants ont transgressé les éléments de la signification lexicale du mot, reconnaissant ainsi l'importance du savoir-être dans la construction de leur propre professionnalité. Sur cette trajectoire de confirmation du cinétisme du mot, les discours des étudiants de Master FLE-S nous permettent de constater que c'est plutôt à travers la formation dans les métiers de l'enseignement que la conception normative et opératoire de la professionnalité pourrait changer, évoluer et, de plus en plus, s'humaniser (rapport au savoir-être).

\section{Des savoirs d'action à la construction d'images identitaires : deuxième démarche théorique et méthodologique}

Afin d'explorer les dimensions dites instrumentales et rationnelles qui côtoient la professionnalité, notre but est désormais de procéder à l'analyse des images identitaires liées aux savoirs d'action mobilisés par les futurs diplômés en FLE-S. Notre regard est toujours centré sur les discours des étudiants, mais cette fois-ci, nous établirons deux catégories d'étude différentes : le regard du public natif et du public non-natif. Les théories sur lesquelles s'appuie ce deuxième volet de notre travail s'associent à l'identification de représentations de par l'Analyse du discours et mettent l'accent sur le repérage de savoirs d'action (SA). Le discours sera ici entendu comme une pratique de construction de soi et de la réalité qui entoure le sujet parlant. Les savoirs sont ici définis du point de vue de Sperber (1996) comme «des représentations énoncées» et leur transmission comme "une contamination des idées ». Les SA sont ainsi « des énoncés (une activité discursive, à une «mise en mots») relatifs à la génération de séquences actionnelles, construites et considérées comme efficaces par les acteurs » (Barbier et al, 2004 : 99). Dans notre cas, les futurs professionnels en FLE-S mobilisent des savoirs d'action à partir de leur propre vécu et leur pratique en enseignement. 
Les travaux sur les SA s'appuient sur les postulats théoriques de la Didactique Analytique (DA). Cette théorie s'inscrit dans une perspective applicationniste qui permet d'avoir une réflexion, comme nous venons de le dire, sur les savoirs d'action mobilisés dans la pratique enseignante (implicationnisme), la pratique enseignante étant ainsi le point de départ et le point d'arrivée de la didactique. La DA se situe à l'interface de la linguistique théorique et la didactique des langues étrangères et secondes, c'est pourquoi, elle se positionne comme une nouvelle perspective pour aborder les pratiques sociales. Elle tient compte d'une perspective praxéologique, voire la théorie de l'action humaine et de l'analyse et la théorisation des pratiques, ancrées à la programmation des pratiques comme hypothèses d'action (Argyris et Schön, 1996 dans Barbier et al, 2004).

Vers quels savoirs d'action? Nous estimons indispensable d'expliquer le type de savoirs auxquels nous ferons référence dans ce travail et de justifier ainsi notre choix. Nous avons eu recours aux travaux publiés par Barbier et al. (2004) car les auteurs proposent un essai de caractérisation des savoirs d'action et leur intérêt porte « sur la façon dont l'énoncé du savoir d'action rend compte de soi d'une représentation du sujet-auteur de l'énoncé, soit de son environnement ou encore de son activité » (Barbier et al, 2004 : 229).

Dans leur rapport, les auteurs ont établi trois types de savoirs d'action, chaque groupe porte un certain nombre d'orientations et chacun remplit une fonction. Parmi ces savoirs, nous trouvons, les savoirs d'action de positionnement, de process et d'environnement. Dans ce travail, nous allons nous centrer sur l'analyse de savoirs d'action de positionnement. Selon Galatanu et Barbier, les savoirs d'action de positionnement sont liés au bon geste professionnel et ils ont un aspect opératoire. Ainsi, l'énonciateur montre sa conviction et agit en fonction du développement d'une bonne pratique. Ce type de savoirs tient aussi compte de l'aspect culturel lorsque l'énonciateur s'appuie sur des conceptions acquises autour de son métier et dans son exercice professionnel.

De même, les savoirs de positionnement renvoient à deux logiques identitaires, à savoir, les individuelles et les collectives. Et, ils remplissent notamment trois fonctions identitaires : la fonction de construction identitaire (FCI), d'affirmation identitaire (FAI) et de consolidation identitaire (F.CONSO.I). La FCI fait référence aux énoncés « où l'action permet à l'auteur de mieux se positionner par rapport à l'exercice du métier, d'autant plus qu'il est « débutant»" (Barbier et al, 2004 : 233) ; l'enseignant est ici amené à faire un retour rétrospectif sur son agir et il travaille sur le perfectionnement de sa pratique.

Dans la FAI s'examinent les énoncés qui portent « une affirmation de la position individuelle de l'énonciateur [...] ou bien une affirmation de la position d'un groupe professionnel auquel il appartient » (Barbier et $a l, 2004: 233$ ). La fonction de consolidation identitaire, celle que nous avons identifiée comme «F.CONSO.I» cible l'analyse « des doutes, des inquiétudes et des hésitations » exprimés dans les énoncés et qui démontrent que l'action est toujours en « voie de perfectionnement» (Barbier et al, 2004 : 234). Au vu de ces remarques, nous passons au repérage et à l'analyse des savoirs d'action inscrits dans les discours des étudiants enquêtés.

\subsection{Les savoirs d'action : traitement et analyse des données}

Notre travail a deux étapes obligatoires, dans la première étape, il s'agit de faire le repérage des savoirs d'action (SA) et dans la deuxième, d'identifier les images identitaires pour enfin analyser comment celles-ci forgent, influencent ou transforment les représentations de la professionnalité chez un public de 120 étudiants natifs et non-natifs suivant un Master I et/ou II en FLE-S dans un contexte universitaire français. Les SA sont extraites d'un 
questionnaire cette fois-ci d'explicitation de représentations adapté par nos soins et proposé par Galatanu dans le cadre d'un programme de Master II, du parcours professionnel FLE-S à l'Université de Nantes (France). Le questionnaire que nous avons adapté est ainsi divisé en trois grandes parties. Cependant, pour la présente étude, nous allons nous limiter à l'analyse de cinq thématiques du questionnaire suivies de questions ouvertes.

Les thématiques et les questions seront présentées ci-dessous et constituent dans l'ensemble un type d'image identitaire. Dans chaque analyse, nous cherchons à identifier les logiques identitaires existantes et les formes de manifestation des différentes fonctions identitaires, pouvant aussi s'articuler ou se renvoyer l'une à l'autre. Il est important de rappeler qu'au sein des corpus recueillis dans les 120 questionnaires d'explicitation de savoirs diffusés et ainsi, que pour chaque question proposé (5 questions), nous avons sélectionnés les extraits autour desquels les images en question se mobilisaient le plus et ces extraits représentent également une forte majorité des réponses données par les étudiants à chaque question. Par ailleurs, la sélection des extraits présentés plus bas est issue d'un travail rigoureux de classement de l'ensemble des réponses attribuées à chaque question et renvoyant ainsi à une image identitaire en particulier. Nous précisons aussi que n'avons apporté aucune modification grammaticale aux extraits cités.

\section{Thématique 1 : Image de l'étudiant face au métier d'enseignant}

Question : Que représente pour vous, dans votre vie, "le métier d'enseignant »? Corpus originaux sélectionnés :

«Une représentation en évolution : avant il détenait le s, qui nécessite que l'on avoir, maintenant il doit peu à peu se fondre dans le groupe. C'est un métier qui a du sens, que l'on choisit par vocation, parce que l'on veut se rendre utile et être actif. C'est une mobilisation à plein temps, qui nécessite que l'on donne de soi. » (M2 N : LC vers LI/ FAI + F. CONSO. I)

«C'est le métier sacré et vraiment respectable. En Thaïlande, le professeur est considéré comme le 3e parent (qui guide, accompagne les apprenants pour atteindre à leur but). » (M2 NN : LC/ FAI)

Analyse fournie : Les savoirs d'action de positionnement repérés répondent à deux logiques identitaires, à savoir, l'individuelle (LI) et la collective (LC). Or, dans certains cas, il $\mathrm{y}$ a un va-et-vient entre la LI et la LC, c'est-à-dire que le point de départ est soit l'individu, soit le groupe social. Quant aux fonctions identitaires, nous constatons que tous les énoncés possèdent une FAI, parfois la FAI s'accompagne d'une F. CONSO. I et dans d'autres cas, la FAI engage une FCI.

À travers les énoncés précédents, nous pouvons aussi observer que les étudiants mettent notamment l'accent sur des expériences contournant leurs parcours de formation, leur pays d'origine et leur vie personnelle. Enfin, la représentation du métier d'enseignant comme la « transmission d'un savoir » est quasiment absente et parfois, elle est remplacée par celle de l'aide et de la promotion de « bonnes valeurs » chez les apprenants.

\section{Thématique 2 : Image sur le parcours universitaire et expérientiel}

Question : Considérez-vous que votre parcours de formation vous permette de progresser pour devenir «un(e) bon(ne) professionnel(le) du FLE»? Toutes les étapes de ce 
parcours sont-elles en cohérence avec ce but? Justifiez votre réponse. Corpus sélectionnés :

«Oui, je pense car j'ai fait une licence de langues et un bac littéraire. Je peux donc enseigner le F hors France. Le master FLE n'est pas indispensable car ici, est trop basé sur la linguistique alors que ce n'est qu'un outil de langue. » (M1 N : F CONSO.I)

« Dans l'absolu, non. Le FLE relève d'une approche complètement différente dont seuls mes deux stages ont pu me donner l'idée (et, un peu, le travail réflexif permis par le master 2 pro en FLE que je réalise actuellement, mais seulement après être devenu enseignant de FLE)» (M2 N : FCI)

«Oui, je suis déjà un professionnel de FLE ayant un bagage professionnel, ce parcours de formation et l'expérience de formation me donne occasion d'une remise en question pour revoir les enjeux didactiques d'un autre regard. » (M2 NN : FCI)

Analyse fournie : Un premier constat porte sur le sentiment de manque d'expérience, les étudiants ne se sentent pas rassurés d'enseigner aujourd'hui. En revanche, ils soulignent l'importance d'avoir un bagage professionnel et de faire un travail réflexif sur les connaissances acquises et sur la pratique. En ce sens, l'analyse des différents corpus nous permet de distinguer les trois types de fonctions abordées plus haut, à savoir, la FCI, la FAI et la F.CONSO.I. Dans certains corpus, nous observons que les étudiants introduisent un argument ayant une FAI et ensuite, ils le renforcent avec un autre ayant un FCI (lien avec la pratique, les stages). Nous constatons aussi l'existence d'arguments qui ont une FAI et s'accompagnent des séries argumentatives ayant une F. CONSO. I (en voie de perfectionnement). Pour faciliter le repérage des différentes fonctions, nous nous appuyons sur l'analyse de la formulation de certaines constructions grammaticales (Charaudeau, 1992) : la formule de la négation, le Je «implicite » et « explicite » (voire les pronoms possessifs) et les locutions adverbiales.

\section{Thématique 3 : Image de soi comme enseignant lors des expériences en enseignement en FLE-S}

Question: Quelle image avez-vous de vous-même comme enseignant (e) ou formateur (formatrice) lors de vos expériences en enseignement de FLE/FLS ? Corpus sélectionnés :

«Une image liée à un manque de compétence et d'expérience pour le moment et liée à un manque d'application des méthodes applicatives de la formation. » (M1 N : F CONSO.I)

« - Native ; Maîtrise du sujet ; Bons outils pédagogiques » (M1 N : FAI)

«Au début de mon parcours professionnel, j'avais une très mauvaise image, celle-ci s'est transformée. En confrontant ma pratique avec celle de mes collègues, je me rends peu à peu compte que je peux avoir confiance en moi. » (M2 N : F CONSO.I + FAI)

Analyse fournie: Comme nous l'avons vu dans l'analyse précédente, dans cette nouvelle question, nous faisons encore face aux trois fonctions identitaires, elles se renforcent l'une avec l'autre. À l'égard des énoncés, les étudiants non-natifs (NN) ont une image positive d'eux-mêmes, ils expriment leur dynamisme et leur engagement avec leur métier, avec la langue et la culture française. Les étudiants natifs de leur côté, ont une 
image qui leur rassure grâce à la maitrise de la langue, mais cette image est toujours en processus de construction et peut se voir affaiblie par le manque d'expérience, voire de confiance en soi. Un élément intéressant que nous avons aussi repéré dans notre corpus est qu'il y a une logique identitaire individuelle qui peut devenir collective grâce aux échanges vécus entre pairs.

\section{Thématique 4 : Image de l'étudiant comme futur enseignant ou formateur}

Question : Quelle image avez-vous de vous-même comme futur enseignant (e) ou formateur (formatrice)? Corpus sélectionnés :

« J'en ai une image positive. J'ai l'impression, qu'avec ce que j'apprends, je vois ce que je peux devenir et ce qu'il faut que j'évite. » (M1 N : FAI+ F CONSO.I)

«Un manque de confiance dans ma façon d'expliquer. Une peur de ne pas pouvoir transmettre quelque chose à mes élèves. Toutefois une vraie envie de partager ma langue et ma culture. » (M1 N : F CONSO.I)

« Je ne me suis jamais vraiment posé la question mais j'aimerais bien continuer à enseigner à un public hétérogène et à pouvoir leur transmettre la langue et la culture française sans avoir une grande appréhension de me retrouver devant la classe. Je voudrais aussi me voir confiante que le cours se passera bien. » (M2 N: F CONSO.I)

«Premièrement, j'aimerais bien maîtriser la langue d'une façon intégrale. Je me vois aussi dans le futur comme un enseignant qui s'occupe de faire l'apprentissage un processus intégral dans la langue elle-même à travers différentes méthodologies qui permettent aux élèves d'avoir une connaissance complète et significative pour leur avenir. » (M1 NN : F CONSO.I)

« Un formateur engagé et attentif aux nouveaux outils nécessaires dans l'apprentissage d'une langue. » (M2 NN : FAI)

Analyse fournie : Au sein de ces registres discursifs, nous traitons des formulations répondant aux trois types de fonctions identitaires et nous trouvons des cas où la FAI sert à introduire une F.CONSO.I. De plus, tous nos registres rendent compte de la position individuelle de l'énonciateur ou du groupe social avec lequel il interagit. L'image du futur enseignant ou formateur en FLE-S est, selon le public natif, associée aux groupes de mots qui suivent, l'image positive, le manque de confiance, la peur, l'instabilité d'emploi, alors que, pour le public non-natif, cette image est surtout liée à la maîtrise de la langue, le sens du progrès, la formation continue et le besoin d'adapter l'apprentissage aux besoins des élèves.

\section{Thématique 5 : Image idéale de l'étudiant comme enseignant de FLE-S}

Question : Quelle est l'image idéale de l'enseignant/formateur? Corpus sélectionnés :

«L'image idéale de l'enseignant/formateur est celle de quelqu'un qui est à l'aise avec son savoir. Il doit être attentif et attentionné. C'est aussi celle d'un praticien réflexif qui cherche des solutions pendant et après les cours pour améliorer le contenu et le déroulement. Il doit savoir s'adapter facilement à son public en étant à l'écoute et ouvert sur les autres. Il doit savoir transmettre mais aussi recevoir, donc partager. Il doit être 
passionné, intéressé et intéressant. Il doit être en quelque sorte une référence dans sa matière. » (M2 N : FAI = PP vers $\mathrm{PC})$

«L'image idéale d'un enseignant serait une personne qui soit motivante, patiente et respectueuse avec ses élèves. Une personne qui est aussi bien préparée et qui s'engage à faire de chaque cours, une expérience différente et significative. » (M1 NN : FAI = PC vers $\mathrm{PP})$

Analyse fournie : La fonction identitaire qui prédomine dans les registres précédents est la FAI, parfois la FAI s'articule avec la F. CONSO. I pour aller vers une FCI (savoirs construits à travers le vécu des acteurs). Nous observons aussi l'existence de deux types de positionnement identitaire, à savoir, le positionnement personnel du sujet agissant (PP) et le positionnement personnel du sujet et de son entourage, voire de sa collectivité (PC).

L'image idéale de l'enseignant de FLE-S au regard des informateurs natifs recouvre les représentations suivantes : avoir des nouvelles idées, être attentif, attirer l'attention des apprenants, la transmission d'un savoir de manière ludique et pédagogique et l'ouverture sur les autres. Pour les étudiants non-natifs, un(e) enseignant(e) ou formateur(trice) en FLE-S doit s'adapter aux situations de la vie courante, être patient (e), motiver ses élèves, s'engager avec ses pratiques enseignantes. Il (elle) doit aussi adopter la posture d'un praticien réflexif, capable de reconnaître ses défauts et ses qualités pour améliorer son quotidien professionnel; le futur enseignant est en formation permanente et reconnaît les caractéristiques et les besoins des apprenants.

\section{Conclusion}

La représentation de la professionnalité chez les futurs enseignants de FLE-S se reconstruit proportionnellement à travers l'analyse des éléments apparus dans les discours déclaratifs et au regard des images identitaires invoquées. Même si dans cette étude exploratoire, nous avons juste essayé de trouver quelques indices sur ce qui pourrait être la professionnalité chez un(e) enseignant(e) de FLE-S, des analyses plus approfondies doivent encore se développer.

À la suite de l'application du protocole sémantique de la SPA au mot professionnalité, nous confirmons que dans les discours des étudiants, la représentation conceptuelle du mot est activée au niveau des stéréotypes et des possibles argumentatifs (PA), mais cela entraîne une transgression aux niveaux des DA, c'est-à-dire, des manifestations discursives. Chez les futurs diplômés en FLE-S, la professionnalité se construit des savoir-faire (savoirs opérationnels), des savoirs (savoirs savants) mais elle envisage aussi une ouverture vers la prise en compte du savoir-être. Et, c'est grâce à cette orientation du savoir, mobilisant des valeurs sociales positives, que les étudiants pourront attribuer une dimension réflexive à leurs pratiques enseignantes et construire autrement leur professionnalité.

Les savoirs d'action de positionnement, nécessaires dans la consolidation de la professionnalité des enseignants de FLE-S, ont été soumis à une analyse qui nous a permis de voir comment l'étudiant se positionne dans son discours et au sein d'un contexte professionnel particulier comme celui des pratiques enseignantes. De même, les discours analysés relèvent la présence de valeurs sociales, des émotions, des témoignages, des images de soi et de l'environnement social. 
Dans le cadre de ces réflexions, la construction d'une professionnalité enseignante est fortement liée au parcours de formation de l'étudiant, aux échanges d'expériences et aux réflexions construites au regard de ses pratiques professionnelles. Dans ce sens, les points de vue des étudiants natifs et non-natifs se diversifient, les objectifs d'enseignement et d'apprentissage proposés dans les programmes d'enseignement s'ouvrent à des grands débats et font partie des interrogations d'une recherche scientifique plus large, voire d'une thèse doctorale qui a été menée dans le champ disciplinaire des Sciences du langage au sein du laboratoire CODIRE (désormais fermé) de l’Université de Nantes (France).

La pratique, étant un élément fondamental dans la formation initiale et continue des futurs enseignants, est encore mise en question et semble représenter un besoin du public concerné. En revanche, nos données révèlent que les savoirs théoriques ont encore tendance à monopoliser les programmes de formation en FLE-S en France, écartant la participation des étudiants à une activité professionnelle réelle. Ce constat mérite aussi d'être abordé plus profondément puisqu'il bouleverse les images identitaires de nos énonciateurs.

L'ensemble de données conceptuelles et discursives nous facilite la découverte de dynamiques identitaires qui jouent sur l'individu, sur le choix d'une formation universitaire et sur l'avenir du métier d'enseignant de langues étrangères, en particulier, du FLE-S. C'est pourquoi il est nécessaire d'examiner régulièrement les discours des acteurs de l'enseignement, ceux-ci nous donnent des pistes intéressantes pouvant contribuer à l'évaluation des programmes d'études et d'éducation permanente, à la revalorisation d'une langue, d'une culture, des citoyens, mais surtout d'un métier qui évolue et éduque tout au long de la vie.

\section{Notes}

Les abréviations « $\mathrm{M} 1$ » et « $\mathrm{M} 2$ » représentent l'année de master de l'étudiant(e) ; la consonne « $\mathrm{N} »$ signifie « natif» et la double « $\mathrm{N} »(\mathrm{NN})$ 《 non-natif ». Les abréviations «LI» et « LC » signifient « logique individuelle» et « logique collective ». Comme nous l'avons annoncé plus haut, les dénominations «FAI», «FCI» et «F.CONSO.I» correspondent aux fonctions identitaires identifiées. 


\section{Références bibliographiques}

Anscombre, J-C. et Ducrot, O. (1994). La argumentación en la lengua. Madrid, ES : Gredos.

Argyris, C. et Schön, D. (1996). Le praticien réflexif. Paris, FR : Editions Logiques

Bancel, D. (1989). Créer une nouvelle dynamique de la formation des maîtres. Paris, FR : Ministère de l'Education Nationale.

Barbier, J.-M. (1996). Savoirs théoriques et savoirs d'action. Paris, FR : Presses universitaires de France.

Barbier, J.-M., Berton F. et Boru, J.-J. (1996). Situations de travail et transformation de la formation. Paris, FR : L'Harmattan.

Barbier, J.-M., et Galatanu, O. (1998). Action, affects et transformation de soi. Biennale de l'éducation et de la formation 03. Paris, FR : Presses Universitaires de France.

Barbier, J.-M., Galatanu, O. (dir.) et al. (2004). Les savoirs d'action : une mise en mot des compétences? Action et savoir, Groupe « Savoirs d'action» du CNAM, Paris, FR : l'Harmattan.

Galatanu Olga, «Du cinétisme de la signification lexicale » dans Jean-Marie Barbier (2006). Sujets, activités, environnements. Approches transverses. Paris Presses Universitaires de France, «Education et formation», p. 85-104. Repéré à URL : https://www.cairn.info/sujets-activitesenvironnements--9782130543374-page-85.htm.

Bourdoncle, R. (1991). La professionnalisation des enseignants : analyses sociologiques anglaises et américaines. Revue Française de Pédagogie, 94, p. 73-91.

Bourdoncle, R., et Mathey-pierre, C. (1995). Autour du mot "Professionnalité". Recherche et formation, 19, p. 137-148.

Carel, M. et Ducrot, O. (2005). La Semántica argumentativa: Una introducción a la teoría de los bloques semánticos [La Sémantique argumentative: Une introduction à la théorie des blocs sémantiques]. Buenos Aires, AR: Colihue Universidad.

Charaudeau, P. (1992). Grammaire du sens et de l'expression. Paris, FR: Hachette éducation.

Devoto, G., et Carlo Oli, G. (2002, 2003). Il dizionario della lingua italiana. Firenze, IT: Le Monnier.

De Mauro il dizionario della lingua italiana. (2000). Torino, IT: Paravia.

Dizionario di italino en ligne Treccanit. (1925-2015). Repéré à URL : http://www.treccani.it/vocabolario/professionalita/.

Fleury, S. (2015). Le Trameur. CLA2T/SYLER. Université Sorbonne Nouvelle Paris 3. Repéré à URL : http://tal.univ-paris3.fr/trameur/.

Galatanu, O. (2005). « La sémantique des modalités et ses enjeux théoriques et épistémologiques dans l'analyse des textes » dans J.M. Gouvard (Ed.), De la langue au style. Lyon, FR: Presses Universitaires de Lyon. p.157-170.

Galatanu, O. (2009a). "Semantic and discursive construction of identities". "Europe of Knowledge" [Sémantique et construction discursive des identités]. In Suomela-Salmi, E et Dervin, F. (Eds.). Cross-cultural and Cross-linguistic perspectives on Academic Discourse [Perspectives cross- 
culturelles et cross-linguistiques dans le discours académique]. Amsterdam/Philadelphia, NE, EU: John Benjamin's Publishing Company. p. 275-293

Galatanu, O. (2009b). «L'analyse du Discours dans la perspective de la Sémantique des Possibles argumentatifs : les mécanismes sémantico-discursifs de construction du sens et de reconstruction de la signification lexicale » dans Garric, N. et Julien, L. (Eds.), L'analyse linguistique de corpus discursifs. Des théories aux pratiques, des pratiques aux théories. Clermont Ferrand, FR : Presses Universitaires Blaise Pascal.

Galatanu, O. (2014). "La construction discursive des images et dynamiques identitaires des enseignants et formateurs en FLE/FLS » dans Revue Signes, Discours et Sociétés, 13, Université de Nantes, France. Repéré à URL : http://www.revue-signes.info/docannexe.php?id=3534.

Grande Dizionario della lingua italiana moderna. Vol. III. (1999). Torino, IT : Garzanti.

Lang, V. (1999). La professionnalisation des enseignants. Paris, FR : Presses Universitaires de France.

Lang, V. (2005). Discours sur la professionnalisation des enseignants : cadre d'analyse : Colloque Formation professionnelle des enseignants. Nantes, FR : actes sur Cd-rom. Ministère de l'Education nationale, de l'Enseignement supérieur et de la Recherche, 2007. Cahier des charges de la formation des maîtres en IUFM. Encart B.O n ${ }^{\circ} 1$ du 4-1-2007.

Moreno, J. (2013). «Étude Pilote sur l'analyse des stéréotypes linguistiques du mot « apprendre» et ses représentations chez les enseignants colombiens de FLE » dans Revista de Ciencias Sociales comunicación, cultura y política, 6, p. 11-38. Universidad Escuela de Administración de Negocios. Repéré à URL : http:// http://journal.ean.edu.co/index.php/revistai/article/view/1294.

Moreno, J. (2013-2017). Construction discursive de la professionnalité des enseignants de FLE en France: enjeux identitaires et expérientiels (Thèse de doctorat en Sciences du langage non publiée). Université de Nantes, France.

Putnam, H. (1975). The meaning of " meaning » [La signification de la «signification»]. Philosophophical papers, 2. Cambridge, UK : Cambridge University Press.

Putnam, H. (1990). Représentation et réalité. Paris, FR : Éditions Gallimard.

Sperber, D. (1996). La contagion des idées : Théorie naturaliste de la culture. Paris, FR : Éditorial O. Jacob. 